\title{
Research on Image Enhancement Algorithm with Restrain Noise Function
}

\author{
Yang Xinfeng ${ }^{*}, 1$ and Duan Shumin ${ }^{2}$ \\ ${ }^{I}$ School of Computer \& Information Engineering, Nanyang Institute of Technology, Henan, Nanyang, 473000, P.R. China; \\ ${ }^{2}$ College of Tourism, Kaifeng University, Henan, Kaifeng, 475004, P.R. China
}

\begin{abstract}
In the recent years, the image processing technology has developed quickly with the widening of image application fields. The image processing has been an important and useful technique in the fields of image understanding and computer vision. The image processing is meant to use some algorithms to process a poor-quality image, in terms of the purpose that the processed image has higher definition and more understandability. According to the property of people's vision grey scale and structure, an image enhancement algorithm with restrain noise function is proposed, which defines a new template to calculate gradient. This algorithm can not only enhance the vision effect of the image, but also can restrain noise and enhance the detail signal. The effect of restraining noise is very good. On the other hand, the method can enhance not only the grey scale image, but also the color image, not distorting the color.
\end{abstract}

Keywords: Image processing, Image enhancement, Restrain noise function, Image distortion.

\section{INTRODUCTION}

In forming an image, internal interference and external interference inevitably exist in the transmission, reception and processing of the process, due to the actual performance and the performance of the receiving apparatus through a transmission medium, , and therefore a variety of noise is produced $[1,2]$. This causes the image to show the distribution of the random noise points in black and white, greatly reducing the quality of the image, making the subsequent image processing difficult. Therefore, to reduce or eliminate the image noise is an important research topic. Noise filtering requirements are: filtered image noise with the maintenance of the details of the image. There are many commonly used filtering algorithms, but these algorithms smooth noise points and also lead to blurring and loss of image details. At present, domestic and foreign journals have published many articles regarding the filtered image noise, and have improved methods of the research results [3-7]. these filters in the study on the performance than the traditional filter has improved, but both is no choice or no difference to all input sample filtering. For a noisy image, only a small portion of the pixel is disturbed by the interference noise, the remaining pixels being retained. No selection or some of the original information is bound to damage the image of the filtering process carried out without distinction for each pixel. Therefore, the proposed method is to add another type of image filtering in the filtering process to determine that the first detection of each pixel of the image is contaminated, then to selectively filter, the output results switch between the original pixel gray and the filter $[8,9]$. As it is selectively filtered, to avoid unnecessary filtering operation and blur images, the filtering effect is further improved. However, these methods

*Address correspondence to this author at School of Computer \& Information Engineering, Nanyang Institute of Technology, Henan, 473000, P.R. China; Tel: 13803770071; E-mail: ywind2005@163.com still have some flaws in terms of the judgment and the process of filtering out the noise; such as for a lighter or darker image, false positives and false negative noise are produced, but also there is a significant increase in the computational algorithms, affecting the filtering effect and speed. While these algorithms do not consider the visual characteristic of the human eye, however as the image is filtered to improve the human visual effect or better applied to subsequent image processing, the filtering effect is not very satisfactory.

Image enhancement technology increases theuseful information for the viewer in order to enhance the quality of the image, having better visual effect. For example, the image drowned in the noise is rendered, or the low-contrast image is shown as a high-contrast image, or the highfrequency component of the spatial frequency tone image clarity is enahced, etc. Conventional methods of image enhancement include histogram equalization, homomorphic increase clarity and so on. Histogram equalization method is a technique which is often used. However, for certain frequency gradation smaller area, the contrast is reduced, or this partial information is even eliminated. In addition, the histogram equalization method is sensitive to noise, therefore enhancing visual effect also involves the enhancement of the noise.

\section{DESIGN AND IMPLEMENTATION OF ALGO- RITHMS}

\subsection{LIP Model-Based Image Enhancement Algorithm}

Yuan, Wang et al. [15] presented some LIP model-based image enhancement algorithm which is as follows:

Deng et al. [10] proposed a new method for image enhancement which is Application logarithmic image processing (LIP) model. 
A patch of gray transform function was used to simplify the algorithm; gray-fill function normalization transformation is defined as follows:

$\bar{f}=1-(f / M)$

In which the value of the function $\mathrm{f}$ is defined as gradation $[0, M)$ interval, for 8 bit image, $M=256$. The simplified algorithm can be expressed as:

$$
\begin{aligned}
\log \left(\bar{f}^{\prime}(i, j)\right)= & \alpha \log (a(i, j))+ \\
& \beta[\log (\bar{f}(i, j)-\log (\bar{a}(i, j)))]
\end{aligned}
$$

Wherein $\bar{f}(i, j)$ and $\bar{f}^{\prime}(i, j)$ are the original and processed gradation functions, $\alpha$ and $\beta$ are real numbers, additionally

$\log (\bar{a}(i, j))=\frac{1}{n \times n}\left(\sum_{k=i-n / 2}^{i+n / 2} \sum_{l=j-n / 2}^{j+n / 2} \log (\bar{f}(k, l))\right)$

Then substituting into the formula $\bar{f}(i, j)$ as $f(1)$, and determining the formula (1) as the inverse of $f=(1-\bar{f}) M$ to give $f(i, j)$, the enhanced image can be obtained.

$\mathrm{a} \log (\bar{a}(i, j))$ can enhance the image which can be seen in very dark or very bright areas of the details, $\bar{a}(i, j)$ in the interval $(0,1)$. When $0<a<1, \bar{a}(i, j)^{a}$ as shown in Fig. 1, the non-linear transformation process can extend the dynamic range of a dark area of the image. When $a>1$, the nonlinear transformation process can extend the dynamic range of the image's brighter areas.

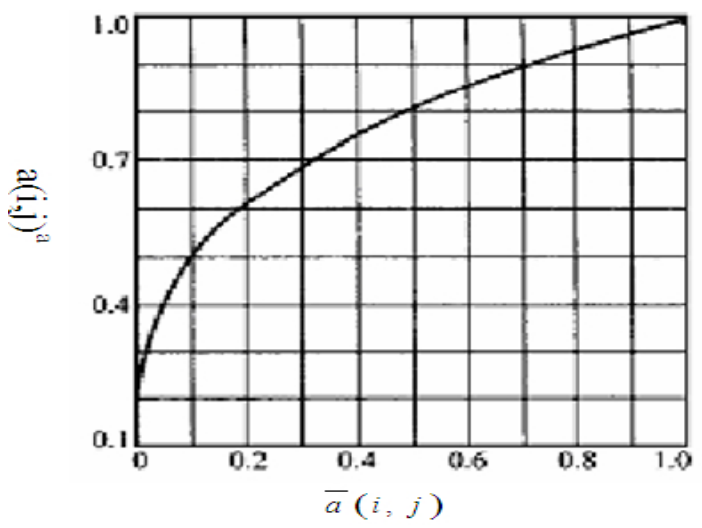

Fig. (1). Nonlinear curve $(a=0.3)$.

Sharpening the image may be obtained by $\beta[\log (\bar{f}(i, j)-\log (\bar{a}(i, j)))]$. When $\beta>1$, the difference between the center pixel and surrounding pixels is a nonlinear amplification. The larger the $\beta$ value, the greater the edges of the image enhancement.
In the actual image processing problems, the original image is often only a small part of a very dark (light) image. The above algorithm can effectively enhance the very dark (bright) contrast areas, but in other parts of the contrast, the image cannot be enhanced. This can be seen by Fig. 1, for $0<a<1$, when the luminance value is small $(\bar{a}(i, j)$ is small), the slope of the transformation curve is large, the contrast of dark areas can be enhanced, when the luminance value is large $(\bar{a}(i, j)$ is large), the slope of the transformation curve is small, so the contrast of the bright areas can neither be enhanced nor be weakened. Similarly, for $a>1$, the contrast of the bright area can be enhanced, and the contrast of dark areas can not be enhanced, and can even be weakened. Therefore, setting a fixed value of $a$ for many images is not suitable.

Another disadvantage of the above algorithm is that it enhances the image details while enhancing the image noise. When $\beta>1$, edges and noise are enhanced.

\subsection{The New Algorithm is Proposed}

Deng et al. [10] manually specified values $a$ and $\beta$ in the calculation of the formula (2).

Yuan, Wang et al. [15] is of the formula (2) into equation (4) to enhance the image.

$$
\begin{aligned}
\log \left(\bar{f}^{\prime}(i, j)\right)= & \log (T(\bar{a}(i, j)))+ \\
& \beta_{s}[\log (\bar{f}(i, j)-\log (\bar{a}(i, j)))]
\end{aligned}
$$

Where they used Prewitt operator orthogonal gradient effect obtained images $I_{g}(x, y)$ and $M A X I_{g}$ the maximum gradient value in the original image, and then $\beta_{s}$ is calculated using the formula $\beta_{s}=\beta_{0}\left|I_{g}(x, y)\right| M A X I_{g}\left(\beta_{0}=3.0\right)$, $\beta_{s}$ effect equivalent to formula (2) $\beta$, Action function $T$ equivalent to formula (2) $a$. They only considered the structural characteristics of the human visual system, without considering the gradation characteristics of the human visual system.

This article also used equations (1), (2), (3) and (1), the inverse of $f=(1-\bar{f}) M$ to enhance the image and took full account of the human visual system, "Weber's Law " or grayscale characteristics and "structure noise mask "and other visual features, to automatically generate the values $a$ and $\beta$ based on the image characteristics.

Specific method is as follows:

According to 'visual system having a structured region at the edge or area smaller than the smoothing noise sensitivity', local contrast of the image increases in detail part of a larger value, smaller in the flat portion, i.e., so that the formula (2) parameter $\beta$ with spatial resolution region is changed. Image gradient reflects spatial resolution of the image, so Yuan, Wang et al. [15] used the orthogonal 
Prewitt operator action resulting gradient images to the original image, since it considers only horizontal and vertical directions, not considering the diagonal direction. The effect is not very good, therefore, the role of a gradient operator is defined to obtain the gradient image in the original image $I_{g}[i, j]$.

Gradient operator is defined as follows:

Horizontal partial derivative :

$P_{0}[i, j]=I[i-1, j]-I[i+1, j]$

Vertical partial derivative :

$P_{90}[i, j]=I[i, j-1]-I[i, j+1]$

$135^{\circ}$ direction of the partial derivative :

$P_{135}[i, j]=2 \times(I[i-1, j-1]-I[i+1, j+1]$

$45^{\circ}$ direction of the partial derivative :

$P_{45}[i, j]=2 \times(I[i+1, j-1]-I[i-1, j+1]$

$I_{g}[i, j]-\sqrt{P_{0}^{2}[i, j]+P_{90}^{2}[i, j]+P_{135}^{2}[i, j]+P_{45}^{2}[i, j]}$

Here, the definition of gradient operator, and more than Prewitt operator consider two orthogonal directions, and its ability to be smoothed noise

Prewitt operator orthogonal sub stronger than some of the behind the experiment illustrates this point.

Taking into account the human visual system, "Weber's Law " or the gradation characteristic, the threshold is calculated (rewritten as formula 10):

$$
g(x)=\left\{\begin{array}{lc}
-0.009549 x^{2}+30 & 0 \leq x \leq 48 \\
-0.000641 x^{2}+0.162794 x+1.662554 & 48<x \leq 206 \\
-0.017493 x^{2}+8.921283 x-1087.463557 & 206<x \leq 255
\end{array}\right.
$$

In order to achieve the same time enhancing the contrast of the dark areas and enhancing the contrast of the brighter area, the formula (2) is required where $\alpha$ is greater than zero in the dark region but less than 1 , while in the brighter region, it is greater than 1 . Therefore, the formula (2) in the $\alpha$ formula (11) is calculated as:

$\alpha=0.79+$ average $/ 142.0$

Wherein: average is a measuring point of the average value of $3 \times 3$ neighborhood.

So in the dark area, the value of average is small, so the value of $\alpha$ is less than 1, conducive to enhancing the contrast of the dark areas; in bright area, the value of average is large, so the value of $\alpha$ is greater than 1, conducive to enhancing contrast in the bright areas. This is more than set a fixed value of $\alpha$ is much better.
In order to achieve enhanced image detail while suppressing noise requirements of formula (2) $\beta$ gray level in sensitive areas of the human eye and the structured area larger, is conducive to enhanced image detail (because the human eye is easy to distinguish their differences), in the human eye is not sensitive to the gray area and smaller flat areas, help suppress noise (because the human eye is not easy to distinguish their differences). Therefore, the formula (2) and $\beta$ formula (13) is calculated by the formula (12).

$\beta=I_{g}[i, j] \times 0.097 /$ threshold

Wherein: threshold is to average as formula (10) calculated out into equation $x, I_{g}[i, j]$ is represented by the formula (8) or (9) calculated gradient values.

So that the same gradation area, threshold is the same value, the point to be detected when the gradient value is relatively large, the value of $\beta$ is large, and the edge information is boosted; when the gradient value is small, the value of $\beta$ is small, conducive to suppress noise. And a gradient value for the same, in different gray areas due to the value of threshold is different from the calculated value average is not the same, so that the obtained values are different $\beta$. In smaller value gradation sensitive eye area threshold, then for the same gradient value, $\beta$ value is large, and boosting the edge information, the person is not sensitive to the value of the eye region threshold gradation is large, for the same gradient value, the smaller $\beta$ values are conducive to suppress noise. This not only takes into account the structural characteristics of the human visual system, but also takes into account the human visual characteristics of gray, making the set fixed value of $\beta$ much better. Because Yuan, Wang [15] $\beta_{s}$ values only consider the gradient value, which are the structural characteristics of the human visual system, and do not consider the grayscale characteristics of the human visual system, so here the calculated value $\beta$ of Yuan, Wang is more reasonable than $\beta_{s}$ value calculated.

The new algorithm is based on the problems of Deng algorithm, combining the visual characteristics of the human eye, $\alpha$ and $\beta$ can be generated automatically, depending on the value of the gray area and gradient values which point to be detected, so that the new algorithm can effectively enhance the most contrasted part of the image, and the noise can be suppressed, which cannot only enhance the gray image, but in the case of enhancing a color image, the effect will be more pronounced.

\section{PERFORMANCE OF THE ALGORITHM IN TERMS OF DE-NOISING}

\subsection{The Traditional Image Filtering Algorithms}

(1) Neighborhood average method 
Neighborhood average method is a local spatial domain approach. For the pixel at position $(i, j)$, gray value $f(i, j)$, smoothed gray value of $g(i, j)$, and the average value of $g(i, j)$ is determined by a number of gray pixels comprising $(i, j)$ neighborhood, i.e., the formula (13) can smooth the gray values of the pixels.

$g(i, j)=\frac{1}{M} \sum_{(x, y) \in A} f(x, y) \quad x, y=0,1,2, \ldots, N-1$

Formula (13) wherein A represents the set $(i, j)$ as the center points in the neighborhood, $\mathrm{M}$ is the total number of pixels in $\mathrm{A}, \mathrm{N}$ is the length or width of the image (in pixels, the same length and width are considered).

Smoothing effect of the neighborhood average neighborhood radius using. The larger the radius, the better the smoothing effect, but the degree of smoothness of the image becomes blur.

Advantage of the neighborhood average method is that the algorithm is simple and involves fast calculation. The main drawback is the noise while reducing image blurring, especially at the edges and details, the larger the neighborhood, the more powerful the fuzzy.

\section{(2) Gauss filter}

Gaussian filter is used as a template of formula (14) shown in the original image convolution operation to achieve the purpose of filtering.

$h=\frac{1}{16}\left[\begin{array}{lll}1 & 2 & 1 \\ 2 & 4 & 2 \\ 1 & 2 & 1\end{array}\right]$

From formula (14), it can be seen that since the right measuring point corresponding to a pixel value of 4 is greater than its neighbor pixels corresponding to the weights, the pixels to be detected are a large role, so its effect is not denoising very well.

\subsection{Method to Evaluate the Effect of De-Noising}

\subsubsection{Objective Evaluation Method}

This method generally uses image PSNR (PSNR) to represent. PSNR is calculated as equation (15) below:

$$
P S N R=10 \log _{10} \frac{255^{2}}{\frac{1}{M \times N} \sum_{i=0}^{M-1} \sum_{j=0}^{N-1}\left(f(i, j)-f^{\prime}(i, j)\right)^{2}}
$$

Description: Formula (15) $M, N$ are the image width and height, $f(i, j), f^{\prime}(i, j)$ are the gray values and after denoising the original image pixel corresponding to the gray value of the corresponding point (Note: where the original image $f$ and $f^{\prime}$ denoised image cannot be the same, otherwise the formula (15) in the denominator would be equal to $0)$.
This method has the evaluation criteria which can be measured to avoid the impact of human factors on the denoising algorithm performance evaluation, with a certain fairness; but it is also hard to find the original image without noise, and sometimes there is no noise-free image this time due to the original image as the reference itself contains noise, while peak signal de-noising method algorithm performance tends to conceal itself.

\subsubsection{Subjective Evaluation}

This approach to human visual perception as a reference, because the end result of the image processing is most people look, which is basically one of the ultimate goal of object of image processing. In this way there are many?, here the following two are introduced:

\subsubsection{Enlarged France}

This method in part of the image is enlarged, so that people see better, mainly in order to measure space-saving layout or the like of the article taken.

\subsubsection{With Noise-sensitive Algorithm to Detect Noise}

There are many algorithms which are more sensitive to noise; these are used to detect if the image contains much noise after de-noising. This may be a viable method of image processing algorithm. For example, Canny edge detection algorithm is very sensitive to noise, so the Canny edge detection algorithm requires first the image denoising, the edge detection and then, if there is no de-noising, direct treatment is carried out to detect images using Canny edge detection algorithm, as it makes it easier to detect the edge image for the noise point.

\section{ALGORITHM PERFORMANCE IN TERMS OF IMAGE ENHANCEMENT}

With a large number of images of the proposed algorithm showing aspects of image enhancement experiments, the results show that the proposed algorithm has a good effect. Lena is now on the original image histogram equalization, Deng method ( $\alpha=0.9, \beta=0.97$; this parameter from the literature [15] gives), [15] method ( $a=0.3, \mathrm{~b}=0.7, \beta_{0}=3.0$ ) for enhanced cultural experiment, the results shown in Fig. 2.

Direct observation of the various enhancement algorithms to enhance image contrast and visual effects is possible from the results in Fig. 2, but if you want to understand the noise enhancement algorithms have also been enhanced, direct observation with the naked eye is not very easy, especially when the picture relatively small size is even more difficult, for example: in the back of the original image in Fig. 4 for the Lena adding white Gaussian noise (mean $\mu=0$ , variance $\sigma=20$ ), but compared with the original image Lena, the naked eye can not see the difference between them (because the image size is relatively small). So Canny edge detection algorithm (without de-noising) is used in Figs. 4-7 on each picture edge detection algorithms to verify the degree of sensitivity to noise; the results are shown in Fig. 3.

On Lena image including Gaussian white noise (mean $\mu=0$, variance $\sigma=20$ ) redoing the above experiment, the 


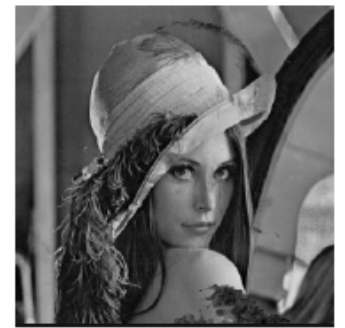

(a)

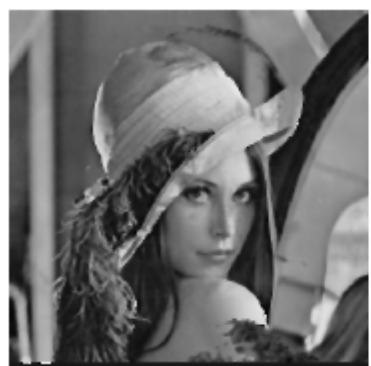

(d)

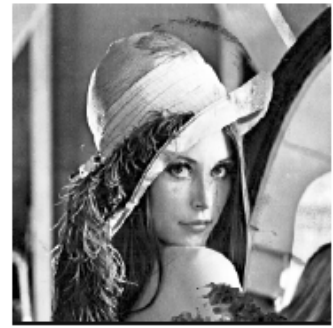

(b)

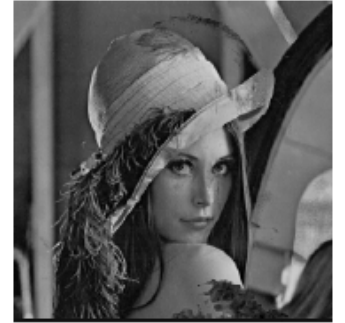

(c)

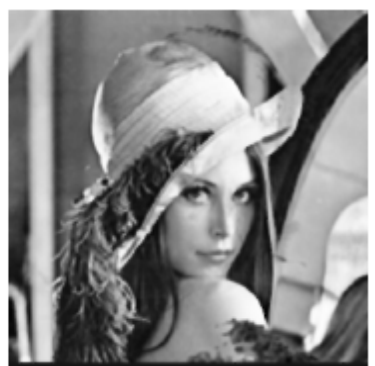

(e)

Fig. (2). Lena original image enhancement algorithm enhanced with a different result: (a) Lena original image (b) Histogram equalization (c) Deng method (d) Literature [15] method (e) The proposed method

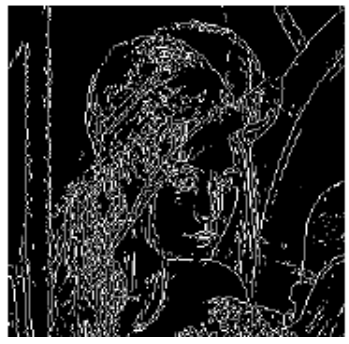

(a)

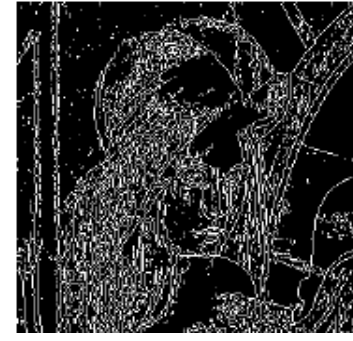

(b)

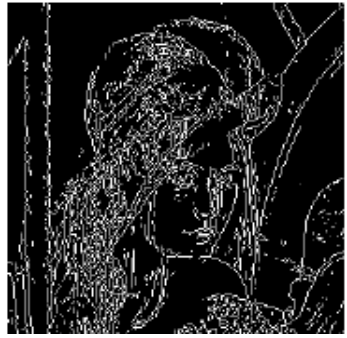

(c)

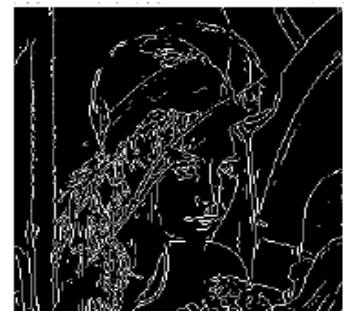

(d)

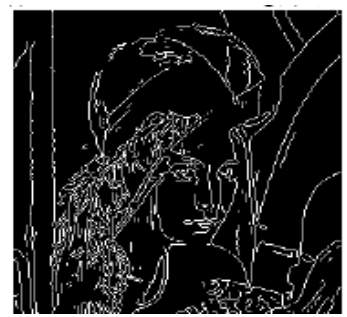

(e)

Fig. (3). Lena original image of each edge of the image enhancement algorithm: (a) Lena original image edge image (b) Histogram edge image (c) Deng method edge image (d) Literature [15] edge image method (e) The proposed method edge image

various parameters of the algorithm remain the same, the results shown in Fig. 4.

In order to more clearly see the situation of the original image and each algorithm, enhanced noise in the picture is extracted from each image enhancement algorithm resulting in a shoulder part of the picture content to be amplified; the experimental results are shown in Fig. 5.
Using Canny edge detection algorithm (without denoising) for edge detection of each picture in Fig. 4, the experimental results are shown in Fig. 6.

From Fig. 2, it can be seen that without noise Lena original image enhancement experiments, the resulting histogram equalization produces most vivid image contrast, the visual effect is best, followed by the proposed method, the literature [15] method surpasses good Deng method some. 


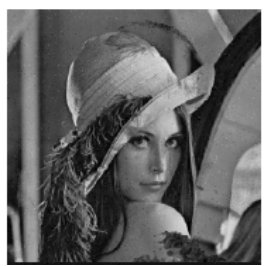

(a)

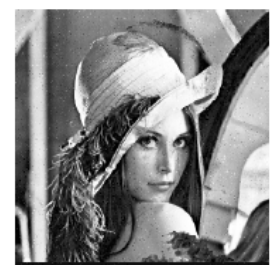

(b)

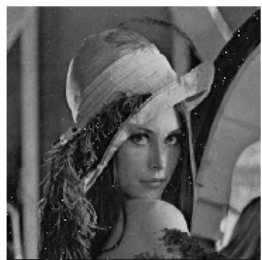

(c)

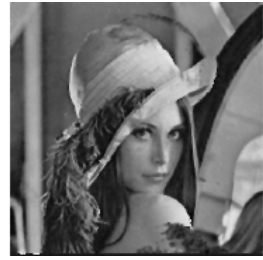

(d)

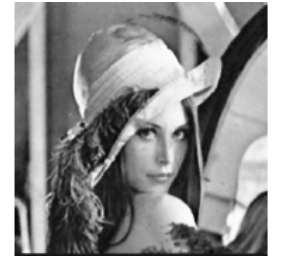

(e)

Fig. (4). Lena original image (noisy) with different enhancement algorithms enhanced results: (a) Lena original image(noisy) (b) Histogram equalization (c) Deng method (d) Literature[15] method (e) The proposed method.

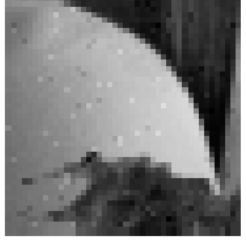

(a)

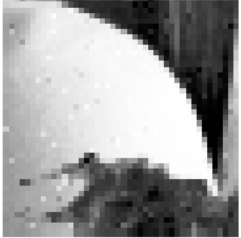

(b)

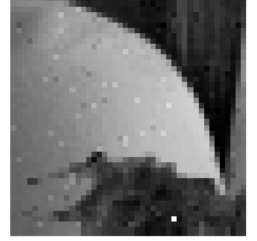

(c)

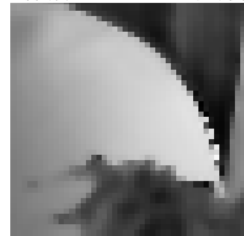

(d)

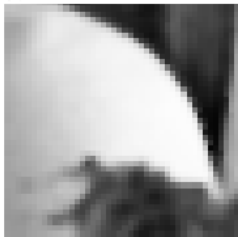

(e)

Fig. (5). Lena original image (with noise) in different parts of the picture enhancement algorithm results: (a) Lena original image (noisy) (b) Histogram equalization (c) Deng method (d) Literature [15] method (e) The proposed method.

From the results of Fig. 3, the edges of the resulting image histogram equalization most rough edges Lena even rougher than the original image, the image on the left edge of the region between the column and the hat. There are many noises, which shows that histogram equalization method is quite sensitive to noise; Deng method edge image obtained is relatively rough, the lower right corner portions of the image have some visible noise points, edge effect and the original image Lena equivalent; the edges of the resulting image [15] are smooth and much delicate, being much less pointed and having less noise, but the image on the left edge of the white pillars of the proposed method is better than some of the rough part, and in the bottom right part of the image, there are some noises clearly visible; the edges of the resulting image are smooth and delicate which shows that the image file is defined by calculating the gradient of the operator than the literature [15] using the Prewitt operator orthogonal better.
Judging from the results of Fig. 4, Fig. 2 can be obtained with substantially the same conclusion, but the right side of the black strip area noise points has been clearly visible in Deng method of the enhanced image, a part of each picture from the shoulder of Fig. 5 point of view, this part of the noise points Deng method is very obvious, because of the higher picture brightness histogram equalization, noise point is not obvious in Deng method, which is consistent with the gradation characteristics of the human visual system (in the white areas of the human eye lowest resolution ), while [15] the proposed method cannot detect the noise points in this section, but there are several obvious particularly bright points (in fact, in the lower right edge of the Figure at the shoulder part of this literature [15] method 2 results also have several bright spots, but are not easy to see due to the small picture size), enhanced over the phenomenon, the literature [15] method has been enhanced over, but the visual effects to enhance the image of the proposed method has not 


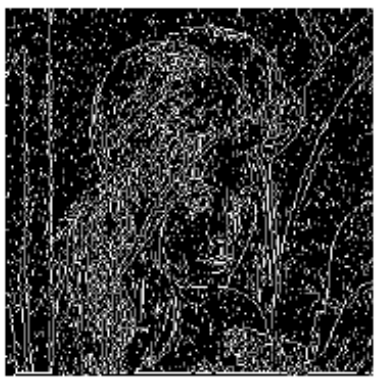

(a)

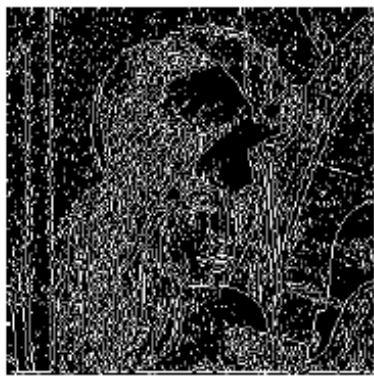

(b)

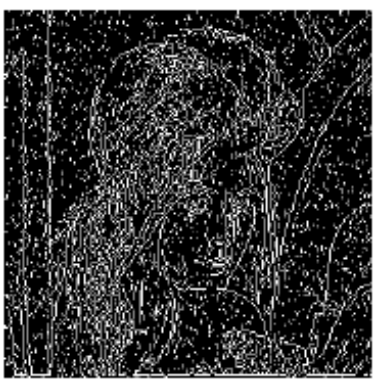

(c)

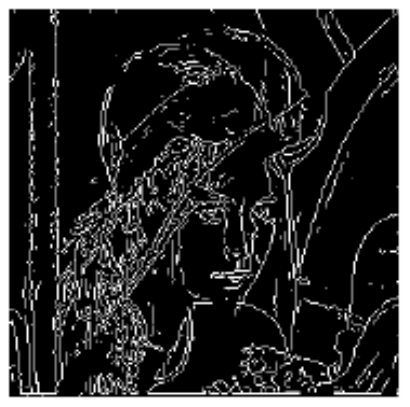

(d)

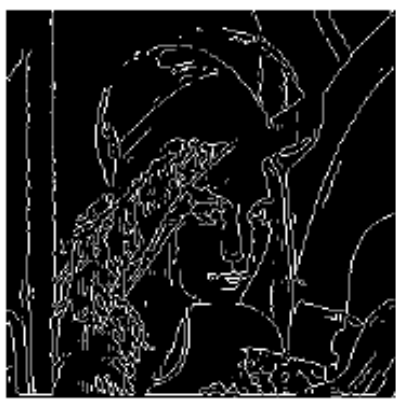

(e)

Fig. (6). Lena original image of each edge of the image enhancement algorithm: (a) Lena original image edge image (b) Histogram edge image (c) Deng method edge image (d) Literature [15] edge image method (e) The proposed method edge image

been a good addition from the upper edge portion of each part of the picture to see this part of the edge of the histogram equalization occurs, Deng methods and literature [15] method in a small jaggies, and the proposed method is not this phenomenon, which further illustrate the text advantage of the method.

From the results of Fig. 6, the edges of the image histogram equalization method and the method of Deng point almost drowned out the noise image, [15] the edge of the image points is not a lot of noise, and Deng than histogram equalization method of noise point is much less, but the resulting image on the left edge of the white pillars and cultural methods than 3 some rough edges, and the noise in the lower right part of the point of the image will be more (compared to Fig. 3 and cultural methods), and text the obtained results are the results of three methods of FIG little difference, but the image on the left edge of the upper part of the column a little rougher, indicating that our method has stronger noise immunity than other methods.

To illustrate the applicability of the proposed algorithm, the use of the proposed algorithm to support the project herein used three images are enhanced, the experimental results shown in Fig. 7:

1 is a port of the original picture, original 2 is a picture of downtown, the airport's original 3 is a picture from the original three can be seen in the upper left and upper right corner down airport runways and enhanced 3 Ping, which is part of the picture in the ring with a spiny, in the original part of the relatively small relatively dark, but it is much too coarse much brighter after enhancement, which shows that the proposed algorithm can effectively sharpen image edges and details information.
Comprehensive comparison of the results of several experiments can be seen that the proposed algorithm can not only effectively enhance image contrast and visual effects, and can effectively suppress noise, you can also sharpen edges and details, the result is an ideal, [15] the method can also enhance the contrast of the image, and also has some ability to inhibit noise, but because they Prewitt orthogonal operator used is not considered in the diagonal direction, easily lost in the diagonal direction of the gradient information is more important they only consider the structural characteristics of the human visual system, without considering the grayscale characteristics of the human visual system, and thus the literature [15] a method for enhancing image contrast and noise suppression capability than the proposed method to be worse, the proposed method can achieve more the desired results, mainly because of the proposed method fully considered the structural characteristics and grayscale characteristics of the human visual system can automatically generate the value $\alpha, \beta$ and use of better operators and other measures in the calculation of the image gradient inevitable result.

\section{PERFORMANCE OF THE ALGORITHM IN TERMS OF COLOR IMAGE ENHANCEMENT}

In the real world, mostly face color image. For color image enhancement, enhanced image detail in noisy environments is one aspect, but compared and grayscale images, color image color information still exist, color image enhancement aims to enhance the image at the same time be able to make the image more vivid detail, color bright but can not bring distortion, for example, the original object becomes red magenta. Therefore, color image processing must correctly select the appropriate color space, as well as deal 


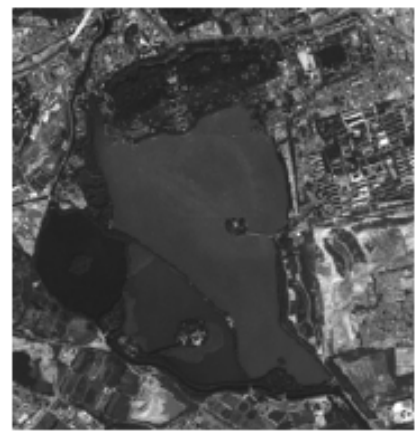

(a) Artwork1

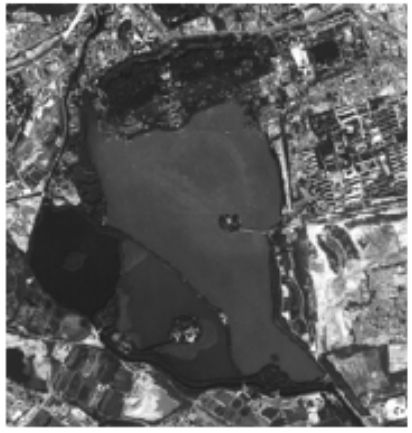

(d) Enhanced map1

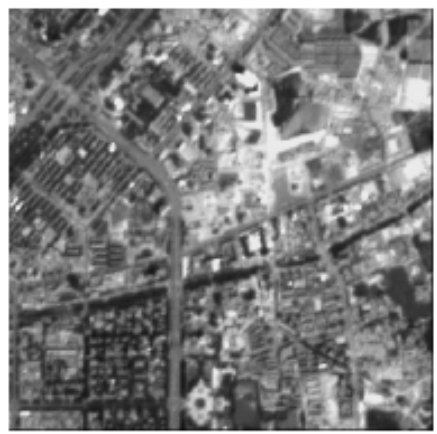

(b) Artwork2

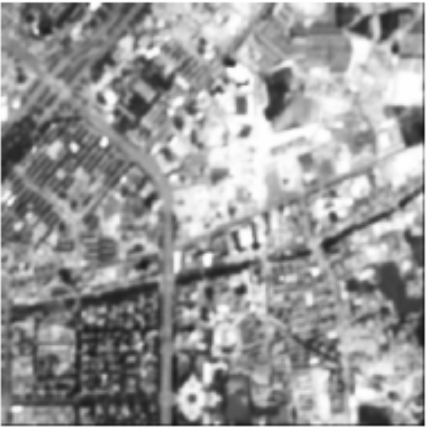

(e) Enhanced map2

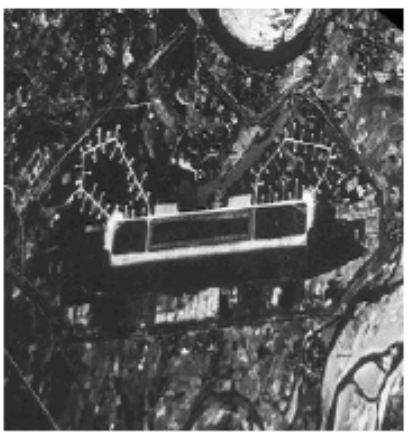

(c) Artwork3

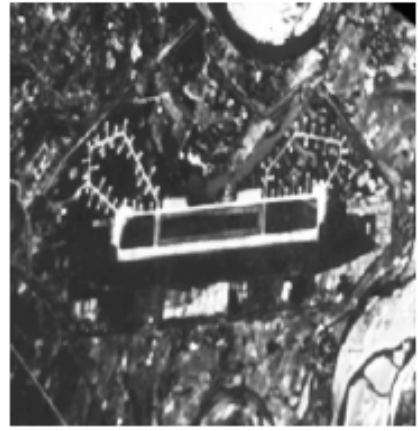

(f) Enhanced map3

Fig. (7). A few examples.

with the relationship between the color components, the paper experimental comparison, corrected the yellow, Wang, Wu et al. [12-14] on the RGB color model bias. The algorithm of this article directly applied to a color image of $\mathrm{R}, \mathrm{G}$, $\mathrm{B}$ component of a color image enhancement, experiments show that the enhanced color image, the details more clearly, more vivid colors and because without causing distortion.

In the color space [11] by use of the color model can be divided into two categories: one is facing, such as monitors, color video camera or a printer hardware. The other is for color-treated for the purpose of applications, such as color graphics animation. The most common color models for hardware devices is the RGB model (Red, Green, Blue), the most commonly used model for dealing with HSI color model (Hue, Saturation, Intensity) . In addition, in the printing industry and television signal transmission, often using CMYK (Cyan, Magenta, Yellow, Black), YUV and Lab color system. One of the most common and most intuitive RGB color model is a model, each pixel of the image has a red, green, and blue gray value, the gray image processing algorithms can be directly applied to the color image of $\mathrm{R}, \mathrm{G}, \mathrm{B}$ component to achieve the purpose of processing a color image.

He, Geng, Zhang et al. [11] pointed out: In the HSI model, component color information has nothing to do with the image, yellow, Wang, Wu et al. [12-14] using the HSI color model image enhancement, also achieved good results However, they noted: "RGB model each component correlation large, HSI model each component associated small." There- fore, Fig. 8 is an image of a component of urban histogram equalization only with HSI model, $\mathrm{H}$ component and $\mathrm{S}$ component unchanged, image enhancement, and then the same image enhancement algorithm using the article, whereby to verify the I component of impact of color information, and the use of HSI model for image enhancement in the presence of problems, but illustrate the effectiveness of the proposed method and the RGB model used in image enhancement aspect of rationality. Finally, the proposed algorithm to other pieces of picture enhancement results.

From the results it can be seen in Fig. 8, the light blue region of the upper left side of the original image, the HSI model I component of histogram equalization later turned into a dark red color and the figure most places have had a distortion, in accordance with He, Geng, Zhang et al. [11] and Huang, Wang, Wu [12-14] of other phrases, only changes I should not produce distortion components, which is why? This can come, Geng, Zhang et al. [11] gives an answer to the conversion formula from HSI model RGB model, this paper gives only within $\mathrm{H} \mathrm{H}\left[0^{0}, 120^{0}\right]$ interval conversion formula:

$$
\begin{aligned}
& B=I(1-S) \\
& R=I\left[1+\frac{S \cos H}{\cos \left(60^{\circ}-H\right)}\right] \\
& G=3 I-(B+R)
\end{aligned}
$$




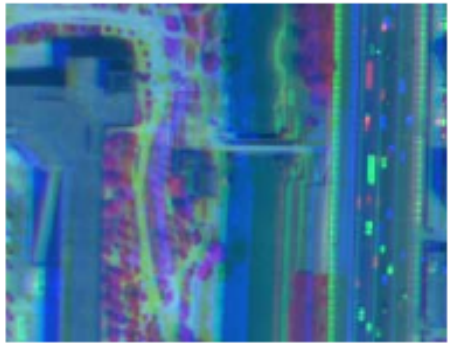

(a)

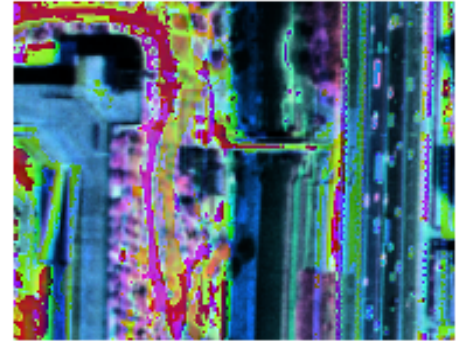

(b)

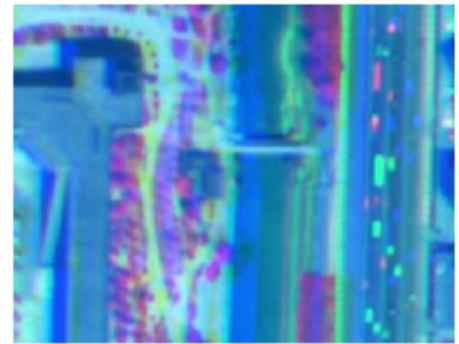

(c)

Fig. (8). Image enhancement for certain urban areas: (a) The original image (b) I component equalization (c) The proposed algorithm.

Table 1. Examples of changing color distortion caused by the I component.

\begin{tabular}{|c|c|c|c|c|c|}
\hline $\mathbf{H}$ & $\mathbf{S}$ & I & $\mathbf{R}$ & G & B \\
\hline 0.684744 & 0.407229 & 138.333333 & 185 & 148 & 82 \\
\hline 0.684744 & 0.407229 & 227.000000 & 48（304） & 242 & 135 \\
\hline 0.684744 & 0.407229 & 227.000000 & 255（304） & 255（291） & 135 \\
\hline
\end{tabular}

Description: The first row in Table 1 is the original image data, the second line is enhanced by overflow after processing, $\mathrm{R}=340>255$, take the overflow value $304-256=48$, $\mathrm{B}=$ $135, \mathrm{G}=227 \times 3-(135+48)=498$, take the overflow value $498-256=242$; the third line is enhanced by a maximum value after processing $\mathrm{R}=304>255$, take the maximum 255 , $\mathrm{B}$ $=135, \mathrm{G}=227 \times 3-(255+135)-291>255$, take the maximum value of 255 .

By the formula (16) to (18) shows that when the $\mathrm{S}$ component and the $\mathrm{H}$ component constant, $\mathrm{R}, \mathrm{G}, \mathrm{B}$ three color varies in proportion to the I component changes, the color is no distortion, but for after I component enhanced, then equation (16) to (18) to calculate R, G, B, the representation may be beyond their range $[0,255]$, then press the overflow regardless of treatment, or by the maximum processing $R, G$, $\mathrm{B}$ ratio to be changed, so that the color will produce distortion. Table 1 shows a specific example:

This shows that when the I-component color enhancement may produce distortion in the practical application of the I component of enhancements, it is difficult to know whether it can cause color distortion, and enhanced components for $\mathrm{H} \mathrm{S}$ components there is a similar problem, in order to for each component are enhanced without causing distortion is not an easy thing to do, and Huang, Wang, Wu et al. [12-14] using the HSI color model image enhancement, also achieved very good results. By the formula (16) to (18) shows that I-component will affect the color information, which is either the same ratio on the color information (Note: no distortion), or changing the ratio of the respective color information (distortion), from Fig. 8, formula (16) to (18) and table 1 that in the HSI model for each component are enhanced rather difficult to control, has a very strong correlation between them, and in the RGB model for R, G, B components, respectively, enhancement, any excessive enhancement will not affect their intermediate the other two components, the correlation between them is not great. The results obtained by the proposed algorithm, colorful, high contrast without distortion, which indicated that the effectiveness of the algorithm, it also shows the use of RGB image enhancement processing model also has its rationality, while expanding the He, Geng, Zhang et al. [11] and Huang, Wang, Wu et al. [12-14] HSI model and the RGB model for understanding and depth of knowledge.

\section{CONCLUSION}

Through the analysis of the text in the image denoising algorithm, grayscale image enhancement and color image enhancement and other aspects of performance comparison shows that the proposed algorithm has a very broad applicability, it can effectively suppress noise, enhancing and preserving image details, not only can enhanced grayscale images, but also can enhance the color image, and will not cause distortion. Proposed algorithm only it will get more satisfactory results, not only with the new gradient operator defined herein and, more important is the use of an algorithm to calculate the threshold of the third chapter defined here is the initial use of the calculation defined threshold method, which a more important role in the calculation of the threshold edge detection method plays.

\section{CONFLICT OF INTEREST}

The authors confirm that this article content has no conflict of interest.

\section{ACKNOWLEDGEMENTS}

This work was financially supported by the Henan Science and Technology Key Project (122102210563 and 132102210215).

\section{REFERENCES}

[1] X. Zhang, D. Chen, and X. Han, "Noise suppression technique based on linear and nonlinear hybrid filter," Computer Engineering and Design, vol. 25, no. 9, pp. 1460-1462, 2004.

[2] X. Han, L. Fan, and J. Li, "An image filtering algorithm based on impulse noise detection," Computer Engineering and Application, vol. 27, pp. 102-104, 2004.

[3] B. Li, T. Peng, and B. Peng, Intelligent Image Processing Technology, Publishing House of Electronics Industry: Beijing, 2004, pp. 52-70.

[4] L. Wu, Data compression, Beijing, 2000, pp. 122-170. 
[5] Y. Zhang, and L. Li, "Multi threshold image denoising method with adaptive wavelet based on GCV theory," Modern Electronic Technology, vol. 11, pp. 62-64, 2007.

[6] F. Bu, and X. Xu, "A SAR image speckle noise filtering algorithm based on wavelet analysis," Journal of Wuhan University, vol. 26, no. 4, pp. 315-319, 2001.

[7] M. Jourlin, and J. C. Pinoli, "A model for log arithmic image processing," Microscopy, vol. 149, no.1, pp. 21-315,1988.

[8] Q. Li, "Research on Algorithm of edge detection in size measurement," Computer Measurement and Control, vol. 12, no. 4, pp. 334-337, 2004.

[9] M. Unser, A. Aldroubi, and M. Eden, "Enlargement or reduction of digital image with minimum loss of information," IEEE Transaction on Signal Processing, vol. 4, no.3, pp. 247- 258, 1995.

[10] G. Deng, L. W. Cahill, and G. R. Tobin, "The study of logarithmic image processing model and its application to image enhance- ment," IEEE Transaction on Image Processing, vol. 4, pp. 506$512,1995$.

[11] D. He, G. Nan, and Y. Zhang, Digital Image Processing, Xi'an, 2003, pp.102-130.

[12] K. Huang, Q. Wang, and Z. Wu, "Multi-scale color image enhancement algorithm based on human visual characteristics," China Journal of Image and Graphics, vol. 8A, no. 11, pp. 1242-1247, 2003.

[13] K. Huang, Q. Wang, and Z. Wu, "Multi scale color image enhancement based on human visual properties," Journal of Circuits and Systems, vol. 8, no. 6, pp. 113-117, 2003.

[14] K. Huang, Q. Wang, and Z. Wu, "Multi scale color image enhancement based on visual characteristics and color space," Chinese Journal of Electronics, vol. 32, no. 4, pp. 673-676, 2004.

[15] X. Yuan, X. Wang, and X. Wang, "An adaptive image enhancement algorithm based on human visual characteristics," Chinese Journal of Electronics, vol. 27, no. 4, pp. 63-65, 1999.

Received: September 16, 2014

Revised: December 23, 2014

Accepted: December 31, 2014

(C) Xinfeng and Shumin; Licensee Bentham Open.

This is an open access article licensed under the terms of the Creative Commons Attribution Non-Commercial License (http://creativecommons.org/licenses/by-nc/4.0/) which permits unrestricted, non-commercial use, distribution and reproduction in any medium, provided the work is properly cited. 